\title{
Fouling mechanism and cleanability of ultrafiltration membranes modified with polydopamine-graft-PEG
}

\author{
Fang Li $\mathbf{i}^{1,2}$, Chunhua Deng ${ }^{1,3}$ *, Chunhui Dư ${ }^{4}$, Bo Yang ${ }^{1,2}$ and Qing Tian ${ }^{1,2}$ \\ 'College of Environmental Science and Engineering, Donghua University, Shanghai 201620, China \\ ${ }^{2}$ State Environmental Protection Engineering Center for Pollution Treatment and Control in Textile Industry, Shanghai 201620, China \\ ${ }^{3}$ Department of Environmental Science, Baylor University, Waco, TX 76798, USA \\ ${ }^{4}$ College of Environmental Science and Engineering, Zhejiang Gongshang University, Hangzhou 310018, China
}

\begin{abstract}
Membrane surface modification via grafting poly(ethylene glycol) (PEG) onto the coated polydopamine (PD) layer is an attractive strategy because it can improve the hydrophilicity of the membrane surface. Sodium alginate (SA), bovine serum albumin (BSA), and humic acid (HA) were used as model foulants to investigate the fouling mechanism and cleanability of modified membranes. The modification narrowed or blocked the membrane pores, which led to a reduction in the permeability of ultrafiltration membranes. A Hermia model was used to explore the fouling mechanism of the modified membranes. PD-g-PEG modified membranes exhibit a lower adsorption for the model foulants and a better cleanability than the unmodified membranes.
\end{abstract}

Keywords: polydopamine, organic matters, PEG, fouling mechanism.

\section{INTRODUCTION}

Because of the pressing demand for purified water, membrane processes, including microfiltration (MF), ultrafiltration (UF), nanofiltration (NF), and reverse osmosis (RO), have received a great amount of attention over the last few decades (Dulebohn et al., 2014; Fane and Fane, 2005; Geise et al., 2010). Membrane fouling, which is responsible for continuous flux decline, is considered to be a major hurdle in the application of membranes for water and wastewater treatment. A large number of studies concluded that most of the fouling resistance was induced by dissolved organic matter in the feed. Consequently, frequent membrane cleaning and, ultimately, membrane replacement are required to maintain the membrane permeability, which increase the operational cost of membrane processes.

Membrane biofouling in water and wastewater treatment, especially irreversible fouling, is usually caused by dissolved organic matter (Mannina et al., 2010). These organic compounds are termed as extracellular polymeric substances (EPS), when they are bound to the flocs, or as soluble microbial products (SMP), when freely suspended in the supernatant. Numerous research studies have been conducted on membrane organic fouling, particularly related to EPS (Sheng et al., 2010; Mannina and Di Bella, 2012). These biopolymers include polysaccharides, proteins, humic acids, nucleic acids, and fatty acids (D’Abzac et al., 2010; Drews et al., 2006).

Membrane surface modification is an effective technology to improve the anti-fouling performance of the membranes (Shannon et al., 2008; Byun et al., 2013). Surface hydrophilization is one of the most attractive methods to reduce membrane fouling because it can decrease the adsorption and deposition of the contaminants onto membrane surfaces. The assumption appears to be reasonable because hydrophilic surfaces are able to attract a layer adjacent to them composed of water molecules

\footnotetext{
* To whom all correspondence should be addressed.

e-mail: lif369@hotmail.com

Received 21 March 2014; accepted in revised form 28 May 2015
}

and prevent hydrophobic foulants from adhering to membranes (Peeva et al., 2012), which leads to a stable and higher water flux at a constant pressure.

The use of PD-g-PEG is currently considered to be an attractive strategy in the membrane surface modification field, because it is able to remarkably enhance the surface hydrophilicity (Zhang et al., 2013; Eshet et al., 2011; Rana and Matsuura, 2010). Recently, some researchers developed a facile, two-step, dopamine-based process to coat a very thin PEG layer onto a membrane surface (Cao et al., 2013; Lee et al., 2007; Lee et al., 2009). A tightly adhesive polydopamine coating layer can be formed by treating a surface with an atris(hydroxymethyl) amino methane hydrochloride (TRIS $\mathrm{HCl}$ ) buffered dopamine solution, regardless of the substrate material (Lee et al., 2008). Moreover, the coating process is simpler and more economical than alternative processes. A variety of membranes, including MF, UF, NF, and RO membranes, have been modified by PD and PD-g-PEG to exhibit a reduced BSA adhesion (McCloskey et al., 2010). In addition, PD and PD-g-PEG modifications are found to influence the pure water flux differently for each membrane, according to the layer thicknesses of the PD deposition; the coated layer thickness resulting from the subsequent PEG-grafting modification was much smaller than the pore size of the MF and UF membranes, but was larger than the pore size of the NF and RO membranes. However, recent reports revealed that membranes modified only by PD-coating did not behave as ideally as expected. Araujo et al. (2012) used PD coating of the membrane and the spacer to enhance biofouling control and found that biofouling was not eliminated. Similar results were obtained by Miller et al. (2012), who found that no reduction in biofouling was observed during longer biofouling experiments with PD-modified membranes, despite the reduced adhesion of BSA demonstrated in shortterm tests. These phenomena have still not been fully explained. Furthermore, the coated PD and grafted PEG layer might be unstable because of the physical adsorption of PEG-derivatized blocks or graft co-polymers on substrates (Wei et al., 2013). In addition, more research work should be conducted to understand the anti-fouling performance of organic foulants of membranes that are surface modified by PD-g-PEG. 
In this paper, PES membranes with a molecular weight cut-off value (MWCO) of 6000 and 20000 were used as virgin membranes to investigate the fouling mechanism and cleanability of the modified membranes after PD coating and PEG grafting. The contact angles were measured after the membrane samples were dipped into the model foulant solution to investigate the adsorption of the organic dissolved matter.

\section{MATERIALS}

\section{Membrane}

Commercial PES UF membranes were provided by Sepro (Oceanside, CA, USA) with nominal MWCO values of 6000 (PES2) and 20000 (PES20) Da. The membrane samples were wetted with isopropanol for $1 \mathrm{~h}$ and then soaked in Milli-Q water overnight prior to use. Dopamine, Trizma $\mathrm{HCl}$, isopropanol, ethanol, bovine serum albumin (BSA), alginic acid sodium salt, humic acid sodium salt (A2158), sodium dihydrogen phosphate, sodium hydrogen phosphate, potassium hydroxide, sodium chloride, and benzoylated dialysis tubing (2,000 NMWCO) were purchased from Sigma-Aldrich (St. Louis, Mo.). A micro BCA protein assay kit was obtained from Thermo Scientific (Rockford, IL), and poly(ethylene glycol) monoamine (5 $000 \mathrm{Da})$ was purchased from JenKem Technology (Allen, TX). The tris buffer (15 $\mathrm{mM}$ ) used in the membrane modifications was prepared by dissolving Trizma $\mathrm{HCl}\left(2.634 \mathrm{~g} \cdot \ell^{-1}\right)$ in Milli-Q water and adjusting the $\mathrm{pH}$ of the resultant solution to 8.8 using sodium hydroxide (Fisher Scientific, Pittsburgh, PA).

\section{Humic acid purification}

Humic acid (HA) was pretreated extensively to remove the fulvic, metal, and ash content, based on the method described by Tang et al. (2007), which is a slightly modified version of the International Humic Substances Society method. Hydrochloric acid was added to HA to yield a solution with a final concentration of $0.1 \mathrm{~g} \cdot \mathrm{m}^{-1}$ and final $\mathrm{pH}$ of 1.0. The suspension was shaken for $1 \mathrm{~h}$ and then centrifuged. The residue from centrifugation was adjusted to $0.1 \mathrm{~g} \cdot \mathrm{m}^{-1}$ at $\mathrm{pH} 13$ with $\mathrm{NaOH}$. The mixture was shaken and then centrifuged. The supernatant was filtered twice through a $0.2-\mu \mathrm{m}$ polyethersulfone filter under a nitrogen headspace. The filtered solution was adjusted to $\mathrm{pH} 1.0$ with 6 $\mathrm{mol} \cdot \ell^{-1} \mathrm{HCl}$ and then settled overnight prior to being centrifuged the next day. The residue was transferred to benzoylated dialysis tubes (molecular weight cut-off of $2000 \mathrm{Da}$, Sigma-Aldrich) and dialyzed in a large MilliQ water bath. The dialyzing water was changed every few hours during the first day and then once each day. Dialysis was stopped when the conductivity of the dialyzing water became lower than $1 \mu \mathrm{S} \cdot \mathrm{cm}^{-1}$. The purified Aldrich humic acid was freeze-dried and stored in the dark at $4^{\circ} \mathrm{C}$.

\section{Membrane modification}

\section{PD modification}

The wetted and soaked membranes were placed onto a clean glass plate with the edges pressed firmly by a square rubber casting ring ( 5 inch side length). Dopamine $(0.4 \mathrm{~g})$ was dissolved in $50 \mathrm{~m} \ell$ of a Tris buffer solution, and the solution was then poured into the casting ring. The membrane was gently rocked for $1 \mathrm{~h}$. The solution was open to allow for airflow over the membrane surface. After reaction, the membrane samples were soaked in ethanol for 10 min to remove the weakly-bound $\mathrm{PD}$, and rinsed in deionized (DI) water for $30 \mathrm{~min}$.

\section{PD-g-PEG modification}

PEG was grafted onto the PD layer to create PD-g-PEG modified membranes. PEG-NH $(M W C O=5000)$ with a $1 \mathrm{~g} \cdot \ell^{-1}$ concentration was dissolved in $50 \mathrm{~m} \ell$ of a Tris buffer solution and heated to $60^{\circ} \mathrm{C}$. This solution was poured into a $150-\mathrm{mm}$ diameter Petri plate, and the PD-modified membrane was then floated, PD modified side down, in the PEG- $\mathrm{NH}_{2}$ solution for $1 \mathrm{~h}$ in an oven at $60^{\circ} \mathrm{C}$. After $1 \mathrm{~h}$, the membranes were rinsed with DI water to remove the loosely connected PEG-NH ${ }_{2}$. The membranes were then stored in a zip-lock bag under dark conditions at $4^{\circ} \mathrm{C}$.

\section{METHODS}

\section{Flux and rejection measurement}

\section{Flux}

The filtration experiments were performed by using dead-end stirred cells (UHF-76, 11.0-cm diameter, MFS, Inc., Japan). During the initial flux measurement of Milli-Q water, the zero time point was set when the Milli-Q water filtration remained approximately stable for over $10 \mathrm{~min}$. Next, the cells were emptied and refilled with a feed solution of $300 \mathrm{ml}$. The stirring rate was set at $300 \mathrm{r} \cdot \mathrm{min}^{-1}$ to minimize the influence of concentration polarization. Filtration was stopped when the retention volume was approximately $75 \mathrm{ml}$. To obtain similar filtration durations across the different membranes, pressure was provided by a nitrogen gas cylinder at $2.90 \mathrm{kPa}(20 \mathrm{psi})$ for the unmodified membranes, $4.35 \mathrm{kPa}$ (30 psi) for the PD-coating modified membranes, and $5.80 \mathrm{kPa}$ (40 psi) for the PES-g-PEG modified membranes. The concentration of the model foulant solution was $100 \mathrm{mg} \cdot \ell^{-1}$. The specific membrane flux was measured as:

$$
J=\frac{V}{t A \Delta P}
$$

where: $V$ is the permeate volume through the membrane with an active area $A$ collected within time $t$ at the trans-membrane pressure $\Delta P$. The relative flux is defined as $J_{\mathrm{s}} / J_{\mathrm{s} 0}$, and $J_{\mathrm{s} 0}$ is the initial specific membrane flux.

\section{Concentration}

Total organic carbon (TOC) analysis using a TOC- $\mathrm{V}_{\mathrm{CSH}}$ system from Shimadzu, Japan, was performed to measure the model foulant concentration. Both TOC and UV/Vis measurements (UV-visible spectrophotometer from Shimadzu, Japan) were conducted simultaneously for the measurement of the HA concentration at $254 \mathrm{~nm}$ and BSA concentration at $562 \mathrm{~nm}$.

\section{Particle size distribution measurement}

To aggravate the fouling effect, calcium chloride was added into an alginate and humic acid solution to create a $\mathrm{CaCl}_{2}$ concentration of $1.0 \mathrm{mg} \cdot \ell^{-1}$. The particle size distribution of the model foulant solution was measured using the dynamic light scattering (DLS) method (Zeta sizer nano ZS, Malvern, UK). The model solution was prepared $1 \mathrm{~h}$ prior to the DLS measurement. 


\section{Membrane cleaning test}

The membrane cleaning test consisted of 3 steps: hydraulic washing, back flushing and chemical cleaning. First, the cell was refilled gently with pure water to measure the flux of the fouled sample. Next, external cleaning was performed by stirring with $150 \mathrm{~m} \ell$ of pure water at $600 \mathrm{r} \cdot \mathrm{min}^{-1}$ for $10 \mathrm{~min}$, followed by the pure water flux measurement. Then, the membrane sample was turned over and a back-flushing process was applied at $2.18 \mathrm{kPa}$ (15 psi) for $10 \mathrm{~min}$. Subsequently, a pure water flux measurement was performed, with the skin layer facing the feed solution. Finally, the sodium hydroxide solution at $\mathrm{pH} \sim 12$ was filtered for 10 min under the corresponding pressure. After each fouling test was finished, the residual solution was removed. The water permeability was tested after chemical cleaning.

\section{RESULTS}

\section{Pure water flux}

Table 1 presents the specific flux values of pure water for modified and unmodified membranes of PES20 and PES2 under the corresponding pressure. Although the applied pressure is known to have a large effect on the flux because of the compressibility of most composite membranes, different pressures were used to determine the permeability of the modified and unmodified membranes, in order to achieve a similar filtration duration for all of the membranes. The membranes were clearly observed to have a lower permeability after the PD and PD-g-PEG modification. To achieve similar filtration durations in the subsequent pore-blocking model analysis, a lower pressure (2.90 kPa; $20 \mathrm{psi})$ was used for the unmodified membrane and a higher pressure (5.80 kPa; $40 \mathrm{psi}$ ) was used for the modified membrane. Despite the PES20 membrane having a slightly larger pore size than that of the PES2 membrane, the water flux reduction for the PES20 membrane after modification was much more remarkable than that for the PES2 membrane. The PES20 membrane displayed a flux reduction of $\sim 80 \%$ for the PD coating modification and $\sim 92 \%$ for the PD-g-PEG modification, while the PES2 membrane displayed $\sim 22 \%$ and $\sim 65 \%$ flux reductions for the $\mathrm{PD}$ and PD-g-PEG modifications, respectively.

\section{Filtration of the model foulant solution}

To characterize the particle size distribution of the organic matter, a DLS measurement was conducted. As the particle size of the macromolecules changed with time, all of the measurements were conducted $1 \mathrm{~h}$ after the feed solutions were prepared. The particle size distributions of the model foulants, i.e., SA, BSA, and HA, are shown in Fig. 1. Due to the effect of calcium on promoting molecular binding and aggregation, SA and HA have the largest mean particle sizes, 138 and $121 \mathrm{~nm}$, respectively. An interesting phenomenon is the bimodal nature of the particle size distribution of BSA. One potential reason for this bimodal nature is the ability of BSA molecules to be structurally rearranged and to agglomerate in an unstable state at $\mathrm{pH} \sim 7$ because the emulsion inversion point of BSA occurs at $\mathrm{pH} \sim 5$ (Huisman et al., 2000). Another possible reason is related to the measurement method, that is, the fraction with a smaller size cannot be detected when the size distribution is very broad. Regarding the SA, many physicochemical studies have revealed that calcium ions induce chain-chain associations among alginate molecules; the structural features involved in this gelation process were proposed in a model called the 'eggbox model' (Li et al., 2007).

The general sequence of the rejection rates of the three model foulants for both the modified and unmodified membranes was: SA $>$ BSA $>$ HA, as indicated in Table 2. This observation is in line with the common view that the membrane with narrower pores is subjected to higher rejection rates. Interestingly, the TOC removal of BSA was higher than that of HA, although the medium particle size of BSA was smaller than that of HA.

For the three model solutions, the relative reductions in flux were different, as shown in Fig. 2. The flux reduction in the BSA solution filtration, in the range of $70-77 \%$ for the corresponding PES20 membranes and 89\%-95\% for the corresponding

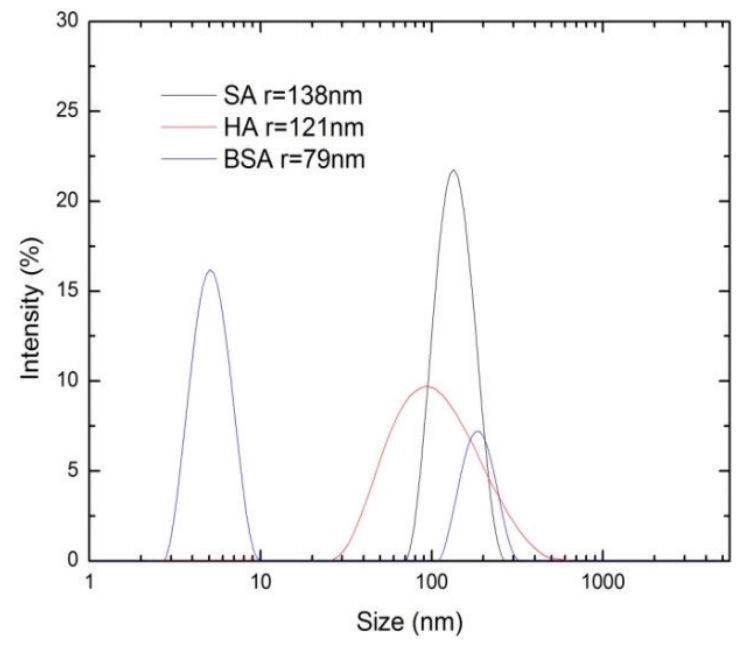

Figure 1

Particle size distributions of SA, BSA, and $H A$ at $100 \mathrm{mg} \cdot \ell^{-1}$ concentration, measured by DLS. BSA was resolved in phosphorus buffer solution with $p H=7, S A$ and $H A$ were resolved in Milli-Q water with $\mathrm{Ca}^{2+} 1 \mathrm{mg} \cdot \ell^{-1}$ and conductivity $1000 \mu \mathrm{S} \cdot \mathrm{cm}^{-1}$.

\begin{tabular}{|c|c|c|c|}
\hline \multicolumn{4}{|c|}{$\begin{array}{c}\text { Table } 1 \\
\begin{array}{c}\text { Flux of virgin membrane. The specific flux of unmodified membrane, PD-coating membrane, and PD-g-PEG membrane } \\
\text { were measured under the pressure of } 2.90,4.35, \text { and } 5.80 \mathrm{kPa}(20,30 \text { and } 40 \mathrm{psi}) \text {, respectively. }\end{array}\end{array}$} \\
\hline \multicolumn{2}{|l|}{ Membrane } & $\begin{array}{c}\text { Specific flux } \\
\left(\ell \cdot \mathrm{m}^{-1} \cdot \mathbf{h}^{-1} \cdot \mathrm{kPa}^{-1}\right)\end{array}$ & $\begin{array}{l}\text { Mean resistance } \\
\left(10^{12} \cdot \mathrm{m}^{-1}\right)\end{array}$ \\
\hline \multirow{2}{*}{ Unmodified } & PES20 & $2.880 \pm 0.087$ & 1.25 \\
\hline & PES2 & $0.509 \pm 0.014$ & 7.07 \\
\hline \multirow{2}{*}{ Coated by PD } & PES20 & $0.547 \pm 0.048$ & 6.58 \\
\hline & PES2 & $0.398 \pm 0.073$ & 9.05 \\
\hline \multirow{2}{*}{ Grafted by PEG } & PES20 & $0.222 \pm 0.071$ & 16.22 \\
\hline & PES2 & $0.179 \pm 0.056$ & 20.11 \\
\hline
\end{tabular}


PES2 membranes, was much lower than that in SA. For the HA solution, a flux decline of less than $10 \%$ was observed. Figures $2 a$ and $2 b$ show the results for relative flux of the SA solution as a function of time, with the highest flux decline observed for the unmodified membrane and the lowest flux decline observed for the PD-g-PEG. The modified membranes of both PES20 and PES2 have lower initial flux values than the unmodified membranes because the pores of the membrane were narrowed by the modification procedure. In all cases of SA solution filtration, a drastic flux decline was observed at the start of the filtration, especially for the unmodified membrane. In the later filtration process, the increase in the flux values slowed down and the fluxes approached a steady-state. This trend in behaviour is much more marked for the PES2 membrane modified by PD-g-PEG, which has the lowest pore size, as shown in Fig. $2 b$. These pieces of evidence indicate that the pore size of the membrane influences the membrane fouling rate remarkably, which is consistent with the report of Luo (Luo et al., 2013).

Comparing the rate of decline of the flux curve between the three model solutions, the effect of particle size was observed. As shown in Fig. 2c, the flux declined linearly with time in the case of unmodified and modified PES20 membranes. For the PES2 membrane samples, the flux curve exhibited 2 stages, the rapid decline stage and the levelling-off stage, similar to the performance of the SA solution. From Figs 2e and 2f, the relative flux of the HA solution decreased linearly with filtration time. The rate of flux decline for HA is lower than that for SA, but is higher than that for BSA.

\section{Adsorptive fouling}

Table 3 presents the contact angles of the membranes before and after being soaked in the various model foulant solutions of SA, BSA, and HA. The contact angles of the modified membranes are clearly smaller than those of the unmodified membranes, that is, the surface of the modified membranes is more hydrophilic than the surface of the unmodified membranes. Note that due to the smoother and denser surface of the unmodified PES2 membranes, the unmodified PES2 membrane has a larger contact angle than the PES20 membrane. A pronounced decrease in the contact angles was observed after these membrane samples were soaked in model foulant solutions, with the ranking of the decrease following a trend of SA > HA
$>$ BSA. This result indicates that adsorption occurred while the membranes were being soaked in these solutions. The membranes soaked in SA displayed the largest reduction, and those soaked in BSA the smallest reduction, in the contact angle value. This result also implies that the order of hydrophilicity of the model foulants is: $\mathrm{SA}>\mathrm{HA}>\mathrm{BSA}$.

\section{Cleaning efficiency}

To accelerate membrane fouling, $1 \mathrm{mg} \cdot \ell^{-1}$ calcium ions were added to the SA and HA solutions. Calcium ions are able to bind with carboxylic functional groups from both solutions and form bridges between adjacent molecules, enhancing their aggregation and thus influencing the rate of flux decline and enabling the reversibility of fouling and membrane rejection (Han et al., 2012). After each filtration test was finished, a membrane cleaning procedure, including surface flushing, back flushing, and chemical cleaning with a $\mathrm{NaOH}$ solution, was performed. The pure water flux, as shown in Fig. 3, was measured after each step. Based on the comparison of the membrane pore size, the UF membranes with higher MWCO, i.e., PES20, tend to lose more permeability because of pore blockage (due to fouling), which is consistent with many other reports. In addition, membranes with larger pore sizes were also found to obtain higher cleaning efficiencies in most cases. Similar results have also been observed by other researchers (Katsoufidou et al., 2008). It is assumed that for the membranes with larger pore sizes, the higher proportion of resistance is induced by pore blockage or constriction. These macromolecules in larger pores are more readily washed out by hydraulic force than are those stuck in small pores. However, such a conclusion can only be drawn when the interaction between the foulants and the membrane surface is negligible compared to the large difference in the pore sizes.

Moreover, it is apparent that the flux recovery by surface flushing for the SA solution filtration is higher than that for the BSA and HA solutions. The other interesting phenomenon is that the membrane samples in the HA fouling tests can recover more flux after $\mathrm{NaOH}$ solution cleaning. The reason for this phenomenon is that the HA is more soluble in the high $\mathrm{pH}$ solution. Note that the coated PD layer or the grafted PEG layer is stable enough to resist both the hydraulic force and $\mathrm{NaOH}$ solution erosion, as was shown in our previous work (Li et al., 2014).

TABLE 2

Rejection of model foulants by unmodified and modified membranes through TOC measurement (\%)

\begin{tabular}{|l|c|c|c|c|c|c|}
\hline & UM-PES2 & PES2-PD & PES2-PD-PEG & UM-PES20 & PES20-PD & PES20-PD-PEG \\
\hline SA & 97.2 & 98.6 & 98.6 & 97.0 & 98.1 & 98.2 \\
\hline BSA & 97.9 & 98.5 & 98.1 & 97.5 & 98.3 & 97.9 \\
\hline HA & 90.4 & 93.7 & 95.2 & 82.1 & 93.8 & 95.2 \\
\hline
\end{tabular}

Table 3

Contact angles of membranes before and after the membranes soaked in model foulants with $100 \mathrm{mg} \cdot \ell^{-1}$ for $5 \mathrm{~h}$, by captive bubble measurement of oil-in-water

\begin{tabular}{|l|c|c|c|c|c|c|}
\hline & \multicolumn{3}{|c|}{ PES2 } & \multicolumn{2}{c|}{ PES20 } \\
\cline { 2 - 7 } & Unmodified & PD coating & PEG grafting & Unmodified & PD coating & PEG grafting \\
\hline Clean & $44.2 \pm 6.2$ & $26.0 \pm 5.3$ & $25.1 \pm 2.7$ & $40.7 \pm 7.9$ & $25.6 \pm 6.1$ & $22.7 \pm 3.8$ \\
\hline SA fouled & $29.1+5.0$ & $22.2+3.6$ & $21.0+4.1$ & $27.5 \pm 5.5$ & $20.7 \pm 2.7$ & $19.1 \pm 2.4$ \\
\hline BSA fouled & $36.2 \pm 3.9$ & $30.5 \pm 2.8$ & $29.2 \pm 3.3$ & $37.6 \pm 4.1$ & $29.0 \pm 2.0$ & $31.7 \pm 4.2$ \\
\hline HA fouled & $29.0 \pm 6.6$ & $28.1 \pm 5.7$ & $29.6 \pm 3.1$ & $31.0 \pm 4.0$ & $27.1 \pm 2.7$ & $23.6 \pm 2.9$ \\
\hline
\end{tabular}



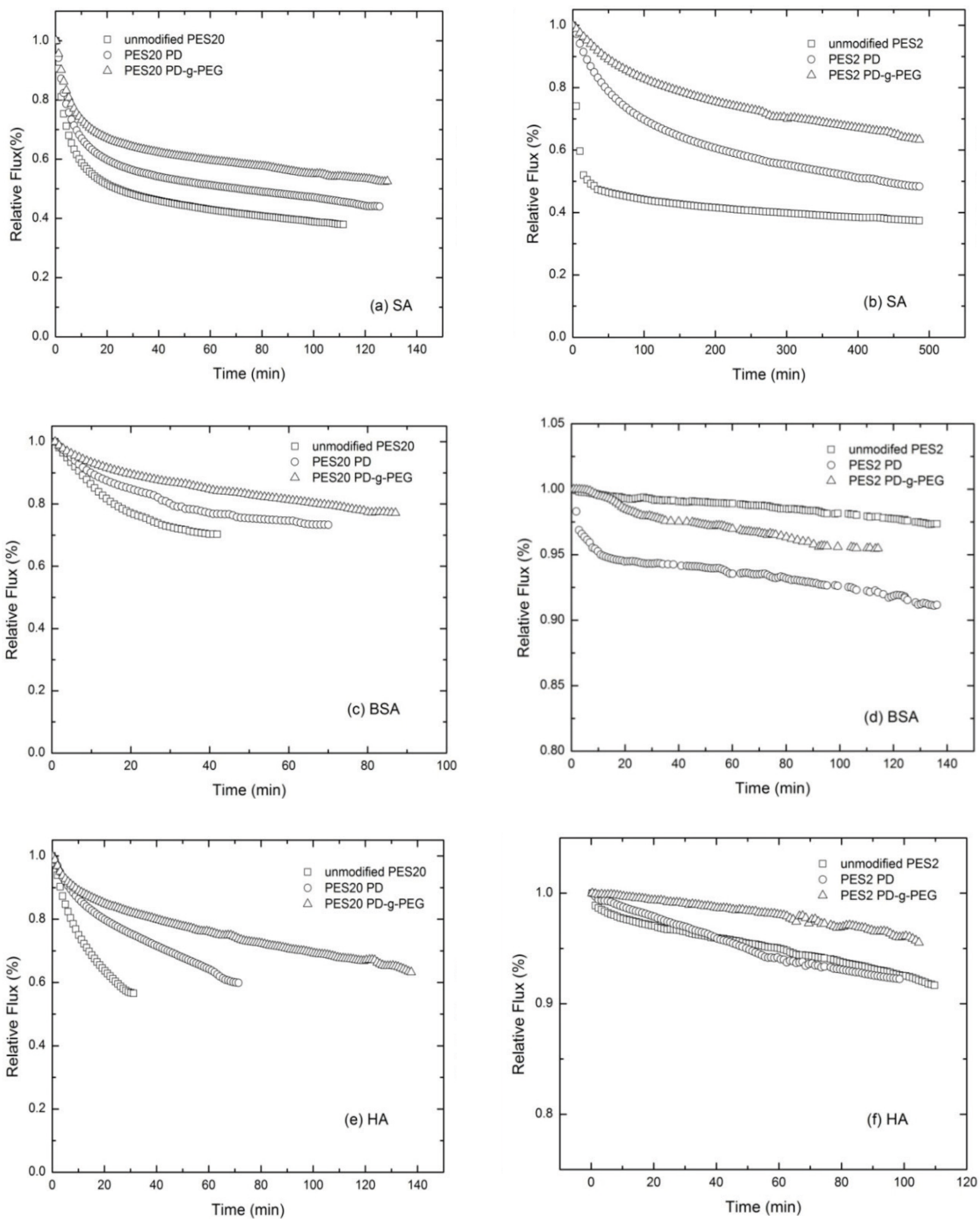

Figure 2

Relative flux J J J versus time during dead-end filtration: (a) unmodified and modified PES20 membranes; (b) unmodified and modified PES2 membranes; (c) unmodified and modified PES20 membranes; (d) unmodified and modified PES2 membranes; (e) unmodified and modified PES20 membranes; ( $f$ ) unmodified and modified PES2 membranes. 


\section{DISCUSSION}

The results of the pure water flux measurement seem to lead to increased flux reduction for the UF membranes with larger pore sizes after surface modification. This result is consistent with McCloskey's report (McCloskey et al., 2012), as a small reduction in the flux for the UF membranes was observed in our work. The
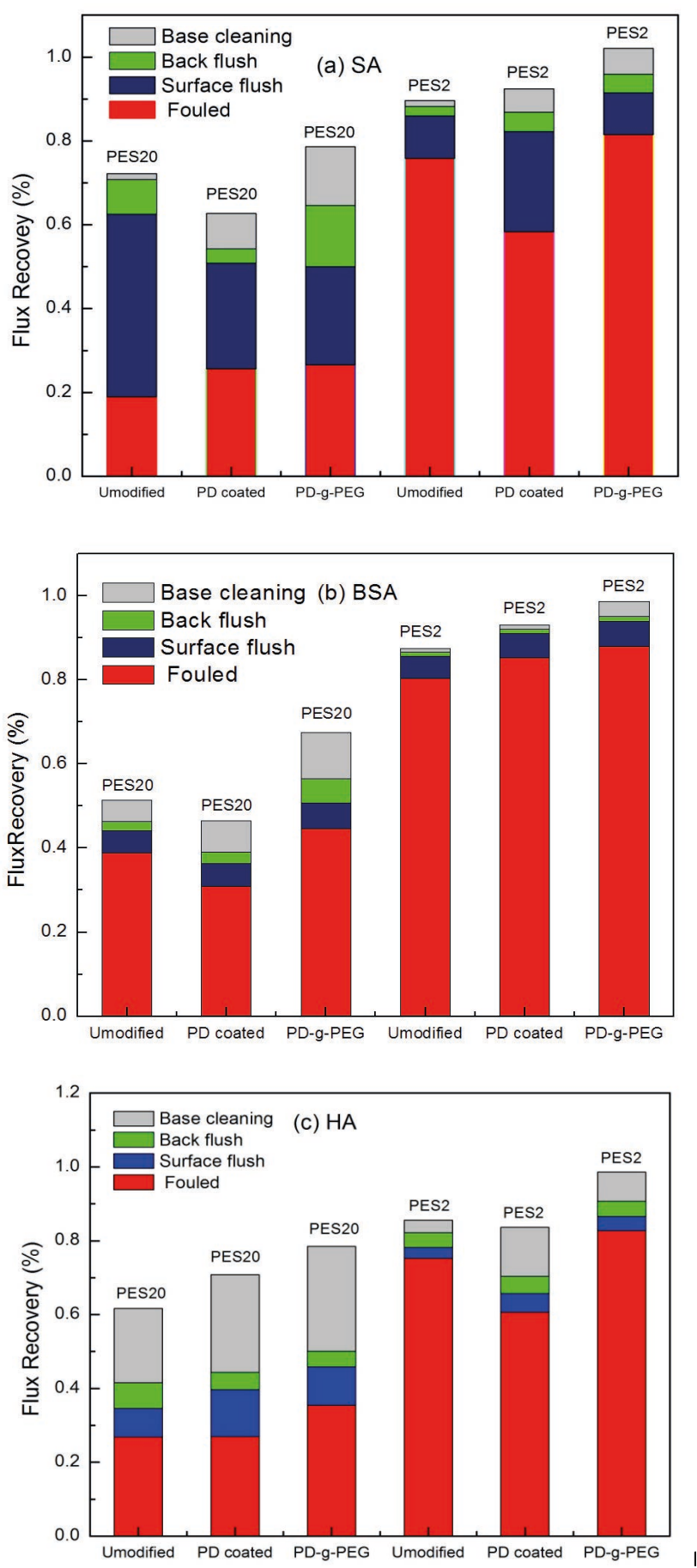

Figure 3

Flux recovery after 3-step membrane cleaning for the model foulants: (a) $S A$, (b) BSA, and (c) HA. The flux was measured with Milli-Q water under a pressure of $2.90 \mathrm{kPa}$ (20 psi) for unmodified membrane, $4.35 \mathrm{kPa}$ (30 psi) for PD-coating membrane, and $5.80 \mathrm{kPa}$ (40 psi) for PD-g-PEG membrane, respectively. assumption for the flux reduction is that PD and PEG are deposited conformally onto the membrane surface and pore structure to constrict the pores or bridged over the membrane surface to block the pores. The TOC removal for BSA was higher than that for HA, despite the median particle size of BSA being smaller than that of HA. This result is in disagreement with the general view that larger particles are rejected with higher probability. This behavior may be attributable to the fact that the real average particle size of the BSA is larger than the measurement result because of the details of the DLS method. The other assumed reason is that the BSA molecules exhibit a stronger charge performance and the modified membranes are negatively charged.

To further investigate the fouling mechanism in the unmodified and modified membranes, the relative fluxes, as a function of time, and Hermia model fitting were plotted. Hermia (Hermia et al., 1982) proposed an empirical model for dead-end filtration under constant pressure, which can be described by Eq. (2). Based on Hermia's filtration law, there are 4 types of fouling mechanisms: complete blocking, intermediate blocking, standard blocking, and cake layer blocking. The type of fouling mechanism considered depends on the value of the dimensionless parameter, $n$.

$$
\frac{\mathrm{d}^{2} t}{\mathrm{~d} V^{2}}=\mathrm{k}\left(\frac{\mathrm{d} t}{\mathrm{~d} V}\right)^{n}
$$

where: $k$ is a constant. Here, $n=2$ for complete pore blocking, which presumably applies to the case of the particle size being larger than the size of the pore opening; $n=1.5$ for standard pore blocking, i.e., pore constriction, which refers to the case that the particles are smaller than the membrane pore size and pore blocking occurs inside the membrane pores; and $n=0$ for cake layer filtration.

Because $\mathrm{d} V / \mathrm{d} t=A J$, it follows that

$$
\frac{\mathrm{d} t}{\mathrm{~d} V}=\frac{1}{A J}
$$

Where $J$ is the permeate flux at time $t$.

$$
\frac{\mathrm{d}^{2} t}{\mathrm{~d} V^{2}}=\frac{1 \mathrm{~d} J}{A^{2} J^{3} \mathrm{~d} t}
$$

To more clearly understand the membrane fouling due to dissolved organic matter, the trend of $\log (-\mathrm{d} J / \mathrm{d} t)$ versus $\log (J)$ is plotted in Fig. 4. In Figs 4a and 4b, two different regions are observed during the filtration process for both the PES2 and PES20 membranes for the SA solution. The first region can be considered to be the pore-blocking condition because the filtration curve shape is a sloped line. The second region corresponds to the steady-state condition, in which the trend of $\log (-\mathrm{d} J /$ $\mathrm{d} t)$ versus $\log (J)$ is presented as vertical straight lines, indicating that the flux varied very slightly with the filtration time. Therefore, the cake layer on the membrane surface was inhibited from forming because of the vigorous stirring. For the BSA and HA solutions during PES20 membrane filtration, as shown in Figs $4 \mathrm{c}$ and $4 \mathrm{e}$, the flux curves were similar to that of the SA solution, that is, they included two regions. In these two cases, the first region can be considered to be the pore constriction condition because of the smaller particle size of the BSA and $\mathrm{HA}$ molecules. In Figs $4 \mathrm{~d}$ and $4 \mathrm{f}$, a vertical line of $\log (-\mathrm{d} J / \mathrm{d} t)$ versus $\log (J)$ was observed because the change in flux with time was quite insignificant given the small pore size of the PES2 membranes and low initial permeate flux.

The features of the organic molecules and the modified surface also affect the membrane fouling behaviour. Calcium ions can bridge more alginate molecules to the deposited ones, 
resulting in the formation of a cross-linked alginate gel layer on the membrane surface (Ye et al., 2005). This process can be used to explain the phenomenon that SA apparently leads to a greater flux reduction compared to BAS and HA. Another reason for the observed phenomenon may be related to the structure of the SA molecules. Because the SA molecules are present as a wrapped sphere in solution with flexible features, they can be easily pushed into the membrane pores under pressure. Thus, the membrane fouling mechanism is not solely determined by the relative particle/pore size. BSA is considered to be a prolate ellipsoid, which has more than 200 positively and negatively charged functional groups, relatively good hydrophilicity, and perfect solubility. Regarding HA, the addition of $\mathrm{CaCl}_{2}$ leads to inter-molecule linkages between the HA molecules. However, these aggregated HA particles still have relatively smaller sizes, resulting in severe pore constriction rather than pore blocking.

Adsorptive fouling was used to simply evaluate the antifouling performance of the coated PD layer and grafted PEG layer. Although short-timescale studies cannot reveal the
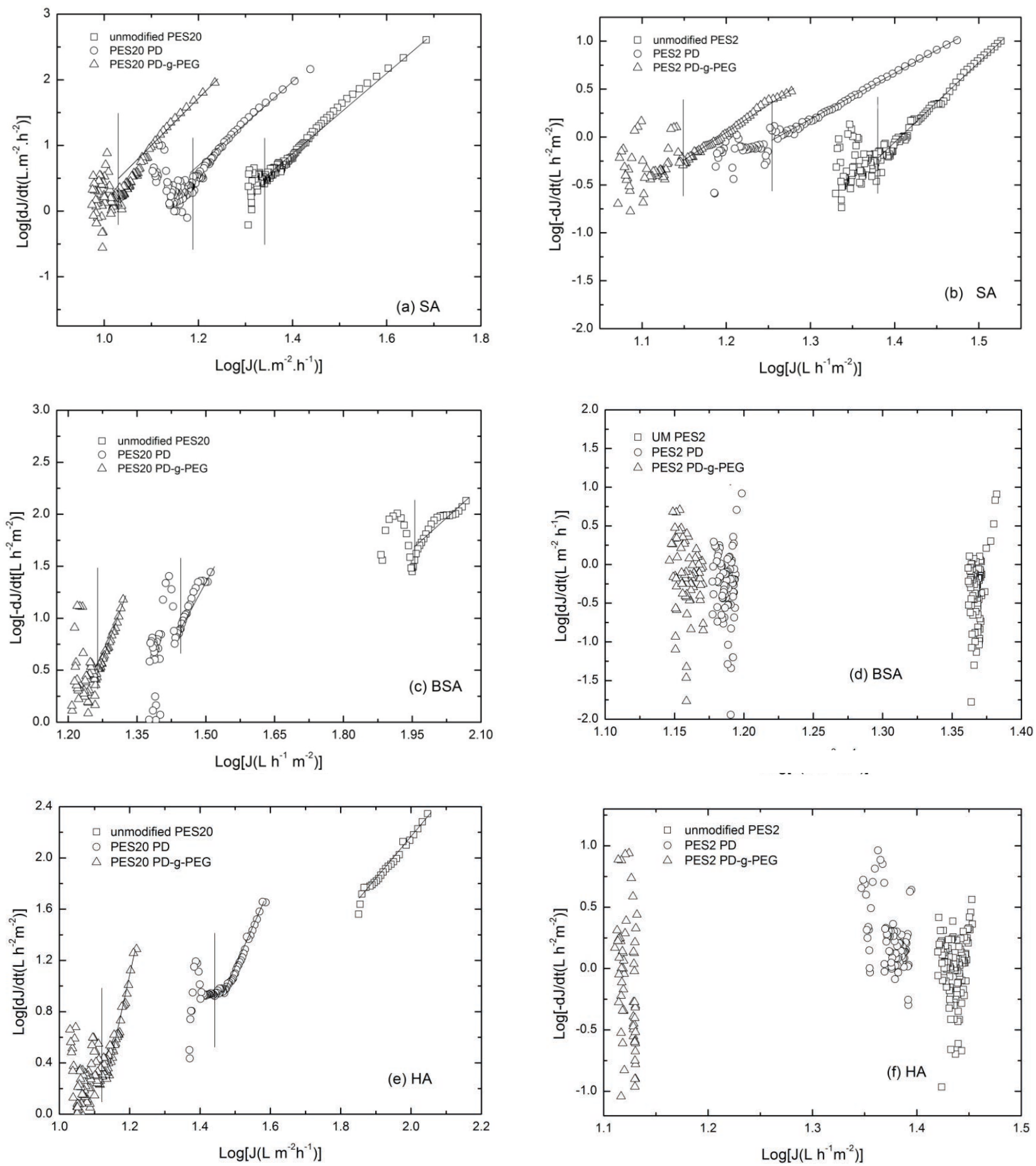

Figure 4

Fouling mechanism analysis basing on log $\left(-d J_{t} / d t\right)$ versus log(J) during dead-end filtration: (a) unmodified and modified PES20 membranes; (b) unmodified and modified PES2 membranes; (c) unmodified and modified PES20 membranes; (d) unmodified and modified PES2 membranes; (e) unmodified and modified PES2O membranes; ( $f$ ) unmodified and modified PES2 membranes. 
complete anti-fouling performance of the modified membranes, short-term organic adhesion tests may be an initial screen for potential antifouling approaches because most organic adhesion occurs within $2 \mathrm{~h}$ (Ridgway et al., 1985). Further, it is quite difficult to translate many laboratory membrane surface modifications to assembled membrane modules that can be used in the long-term bacterial fouling test. There is a need for systematic studies to link the modified membranes to representative results in the practice of long-term anti-fouling studies. An interesting phenomenon observed in this investigation is that the contact angle of the membranes fouled by SA was even lower than that of clean membranes. One possible reason for this phenomenon is that SA is considered to have a more hydrophilic property than PD and PEG. Another possible reason is that PEG molecules grafted on the PD layer form a rough layer, which appears as a 'brush'. The PEG brush is able to reduce the adsorption of organic matter as it prevents the organic molecules from attaching to the membrane surface directly.

In the cleaning tests section, both PES2- and PES20 PD-modified membranes did not exhibit a better cleaning efficiency than the unmodified membranes according to the flux recovery data, although the membrane pores were narrowed after modification. The likely explanation for this is the negativelycharged PD layer. The presence of $\mathrm{Ca}^{2+}$ induces a binding force between the macromolecules, and the PD is stable during SA and HA solution filtration. The hydroxyl groups of the polydopamine layer surface complexed with divalent cations to boost nucleation (Han et al., 2012). The force of the complexed interaction between $\mathrm{Ca}^{2+}$ and the PD layer is strong enough to survive the hydraulic force and chemical reaction. In all cases, the PEGgrafting modified membranes have a higher flux recovery than the other two membrane samples. This phenomenon could be promoted by the neutral and hydrophilic characteristics of the PEG molecules. The 'brush' layer formed by the PEG molecules prevents the foulant molecules from entering into membrane pores or contacting the membrane surface and PD layer directly. Consequently, the complexation was induced by the $\mathrm{Ca}^{2+}$ and not formed on the PD-g-PEG modification membrane surface.

\section{CONCLUSION}

In this study, the fouling mechanism and cleanability of the UF membrane modified by PD-g-PEG were investigated. After the modification, PES20 UF membranes exhibited a greater decrease in permeability than PES2 UF membranes due to the PD and PEG layer that formed on the pore walls resulting in narrower pores. A lower reduction was observed in the relative flux for the modified membranes during model solution filtration. The fouling mechanism mainly depends on the relative relationship of the particle size to the membrane pore size. The shape and structure of the foulant molecules also influences the membrane fouling mechanism. In the adsorption anti-fouling tests, the grafted PEG layer is able to reduce the adsorption of organic matter because it prevents organic molecules from attaching to the membrane surface directly. In the membrane cleaning test, the results demonstrated that the membranes with larger pore sizes presented better cleanability. The PD-gPEG-modified membranes have a higher flux recovery than the other two membrane samples considered.

\section{ACKNOWLEDGEMENTS}

This work was supported by the National Natural Science Foundation of China (Grant No.51478099, 51103130), the
Natural Science Foundation of Shanghai (13ZR1401000), and the Fundamental Research Funds for the central University (223215A3-04).

\section{REFERENCES}

ARAUJO PA, MILLER DJ, CORREIA PB, VAN LOOSDRECHT MCM, KRUITHOF JC, FREEMAN BD, PAUL DR and VROUWENVELDER JS (2012) Impact of feed spacer and membrane modification by hydrophilic, bactericidal and biocidal coating on biofouling control. Desalination 295 1-10.

BYUN YJ, KIM JH and KIM SS (2013) Surface modification of PVDF membranes for water treatment via hydrophilic thermal crosslinking method. Desalin. Water Treat. 51 5371-5378.

CAO Y, ZHANG X, TAO L, LI K, XUE Z, FENG L and WEI Y (2013) Mussel-Inspired Chemistry and michael addition reaction for efficient oil/water separation. Acs Appl. Mater. Interfaces 5 4438-4442.

D'ABZAC P, BORDAS F, VAN HULLEBUSCH E, LENS PN and GUIBAUD G (2010) Extraction of extracellular polymeric substances (EPS) from anaerobic granular sludges: comparison of chemical and physical extraction protocols. Appl. Microbiol. Biotechnol. 85 1589-1599.

DREWS A, LEE C-H and KRAUME M (2006) Membrane fouling-a review on the role of EPS. Desalination 200 186-188.

DULEBOHN J, AHMADIANNAMINI P, WANG T, KIM SS, PINNAVAIA TJ and TARABARA VV (2014) Polymer mesocomposites: Ultrafiltration membrane materials with enhanced permeability, selectivity and fouling resistance. J. Membr. Sci. 453 478-488.

ESHET I, FREGER V, KASHER R, HERZBERG M, LEI J and ULBRICHT M (2011) Chemical and physical factors in design of antibiofouling polymer coatings. Biomacromolecules 12 2681-2685.

FANE AG and FANE SA (2005) The role of membrane technology in sustainable decentralized wastewater systems. Water Sci. Technol. 51 317-325.

GEISE GM, LEE HS, MILLER DJ, FREEMAN BD, MCGRATH JE and PAUL DR (2010) Water purification by membranes: The role of polymer science. J. Polym. Sci. B-Polym. Phys. 48 1685-1718.

HAN K, LIU S, TANG S, LIU X and JIANG S (2012) Polydopamine assisted liquid phase deposition for preparation of zirconia coated column in capillary electrophoresis. Anal. Meth. 4 4193-4197.

HERMIA J (1982) Constant pressure blocking filtration laws - application to power-law non-newtonian fluids. Trans. Inst. Chem. Eng. 60 $183-187$.

HUISMAN IH, PRADANOS P and HERNANDEZ A (2000) The effect of protein-protein and protein-membrane interactions on membrane fouling in ultrafiltration. J. Membr. Sci. 179 79-90.

KATSOUFIDOU K, YIANTSIOS SG and KARABELAS AJ (2008) An experimental study of UF membrane fouling by humic acid and sodium alginate solutions: the effect of backwashing on flux recovery. Desalination 220 214-227.

LEE H, DELLATORE SM, MILLER WM and MESSERSMITH PB (2007) Mussel-inspired surface chemistry for multifunctional coatings. Science 318 426-430.

LEE Y, LEE H, KIM YB, KIM J, HYEON T, PARK H, MESSERSMITH PB and PARK TG (2008) Bioinspired surface immobilization of hyaluronic acid on monodisperse magnetite nanocrystals for targeted cancer imaging. Adv. Mater. 20 4154-4157.

LEE H, RHO J and MESSERSMITH PB (2009) Facile conjugation of biomolecules onto surfaces via mussel adhesive protein inspired coatings. Adv. Mater. 21 431-435.

LI F, MENG JQ, YE JF, YANG B, TIAN Q and DENG CH (2014) Surface modification of PES ultrafiltration membrane by polydopamine coating and poly (ethylene glycol) grafting: Morphology, stability, and anti-fouling. Desalination 344 422-430.

LI L, FANG Y, VREEKER R and APPELQVIST I (2007) Reexamining the egg-box model in calcium-alginate gels with X-ray diffraction. Biomacromolecules 8 464-468.

LUO J, ZHU Z, DING L, BALS O, WAN Y, JAFFRIN MY and VOROBIEV E (2013) Flux behavior in clarification of chicory juice by high-shear membrane filtration: Evidence for threshold flux. $J$. Membr. Sci. 435 120-129. 
MANNINA G, BELLA GD and VIVIANI G (2010) Uncertainty assessment of a membrane bioreactor model using the GLUE methodology. Biochem. Eng. J. 52 263-275.

MANNINA G and BELLA GD (2012) Comparing two start-up strategies for MBRs: Experimental study and mathematical modelling. Biochem. Eng. J. 68 91-103.

MCCLOSKEY BD, PARK HB, JU H, ROWE BW, MILLER DJ, CHUN BJ, KIN K and FREEMAN BD (2010) Influence of polydopamine deposition conditions on pure water flux and foulant adhesion resistance of reverse osmosis, ultrafiltration, and microfiltration membranes. Polymer 51 3472-3485.

MCCLOSKEY BD, PARK HB, JU H, ROWE BW, MILLER DJ and FREEMAN BD (2012) A bioinspired fouling-resistant surface modification for water purification membranes. J. Membr. Sci. 413 82-90.

MILLER DJ, ARAUJO PA, CORREIA PB, RAMSEY MM, KRUITHOF JC, VAN LOOSDRECHT MCM, FREEMAN BD, PAUL DR, WHITELEY M and VROUWENVELDER JS (2012) Short-term adhesion and long-term biofouling testing of polydopamine and poly(ethylene glycol) surface modifications of membranes and feed spacers for biofouling control. Water Res. 46 3737-3753.

PEEVA PD, MILLION N and ULBRICHT M (2012) Factors affecting the sieving behavior of anti-fouling thin-layer cross-linked hydrogel polyethersulfone composite ultrafiltration membranes. J. Membr. Sci. 390 99-112.
RANA D and MATSUURA T (2010) Surface modifications for antifouling membranes. Chem. Rev. $1102448-2471$.

RIDGWAY HF, RIGBY MG and ARGO DG (1985) Bacterial adhesionand fouling of reverse osmosis membranes. J. Am. Water Works Ass.77 97-106.

SHANNON MA, BOHN PW, ELIMELECH M, GEORGIADIS JG, MARINAS BJ and MAYES AM (2008) Science and technology for water purification in the coming decades. Nature 452 301-310.

SHENG GP, YU HQ and LI XY (2010) Extracellular polymeric substances (EPS) of microbial aggregates in biological wastewater treatment systems: A review. Biotechnol. Adv. 28 882-894.

TANG CY, KWON Y-N and LECKIE JO (2007) Fouling of reverse osmosis and nanofiltration membranes by humic acid-effects of solution composition and hydrodynamic conditions. J. Membr. Sci. 290 86-94.

WEI H, REN J, HAN B, XU L, HAN L and JIA L (2013) Stability of polydopamine and poly(DOPA) melanin-like films on the surface of polymer membranes under strongly acidic and alkaline conditions. Colloids Surf. B $11022-28$.

YE Y, LE CLECH P, CHEN V, FANE AG and JEFFERSON B (2005) Fouling mechanisms of alginate solutions as model extracellular polymeric substances. Desalination 175 7-20.

ZHANG W, YANG FK, HAN Y, GAIKWAD R, LEONENKO Z and ZHAO B (2013) Surface and tribological behaviors of the bioinspired polydopamine thin films under dry and wet conditions. Biomacromolecules 14 394-405. 